\title{
Modeling the electron collision frequency during solid-to-plasma transition of polystyrene ablator for direct-drive inertial confinement fusion applications
}

\author{
A. Pineau, ${ }^{1, \text { a) }}$ B. Chimier, ${ }^{1}$ S.X. Hu, ${ }^{2}$ and G. Duchateau ${ }^{1}$ \\ ${ }^{1)}$ Université de Bordeaux-CNRS-CEA, Centre Lasers Intenses et Applications, UMR 5107, 351 Cours de la Libération, \\ 33405 Talence Cedex, France \\ ${ }^{2)}$ Laboratory for Laser Energetics, University of Rochester, 250 East River Road, Rochester, New York 14623, \\ USA
}

(Dated: 4 June 2020)

Within the inertial confinement fusion (ICF) framework, the solid-to-plasma transition of the ablator arouses increasingly interest, in particular due to the laser-imprint issue. Phase evolution of the ablator is linked to the evolution of the electron collision frequency which is of crucial importance since it drives electron heating by laser energy absorption, and lattice-ion heating due to collisions between electrons and the lattice-ion system. Thus, an accurate description of electron collisions over the whole temperature range occurring in ICF, starting from a few tens of kelvins (solid state) up to tens of millions of kelvins (plasma state), is necessary. In this work, polystyrene ablator is considered and a model of chemical fragmentation is presented to describe the heated polystyrene evolution. Electron collisions are described by electron-phonon collisions in the solid state, and by electron-ion and electron-neutral collisions in plasma state. An effective electron collision frequency valid over the whole range of temperatures reached in ICF experiments is established and discussed. Thermal conductivity is also deduced from collisions in the plasma state and shows a good agreement with the one evaluated by ab initio calculations.

\section{INTRODUCTION}

To achieve controlled thermonuclear fusion, direct-drive inertial confinement fusion (ICF) has been intensively pursued over the past decades $1-4$. This process consists of focusing several intense laser beams onto a spherical target in order to compress it to reach temperature and density conditions that are required to generate fusion reactions. The target is usually composed of a cryogenic deuterium-tritium (DT) shell filled with gaseous DT. This cryo-DT shell is covered with an ablator that is usually made of polystyrene ${ }^{5}$. Upon the laser flux, the solid polystyrene layer absorbs the incident energy laser, is converted to a plasma, and is removed from the target leading to the DT compression through the so-called rocket effect. However, the overlapped laser beams can induce spatial variations of intensities on the ICF target surface bringing about a non-uniform ablation of the ablator. This is the so-called laserimprinting process ${ }^{6}$. If the target ablation is non-uniform, perturbations in density and pressure can be induced, becoming the seed of the Rayleigh-Taylor instability. As a consequence, ICF target performances are drastically reduced ${ }^{7} \sqrt{9}$ because of these spatial perturbations. Despite this, the ablation process is partially described in the current ICF hydro-codes where materials are initially assumed in plasma state but the solid-toplasma transition is generally neglected. As a result, a factor of two was observed between experimental measurements and hydrodynamic simulations of shock velocity modulations 10 .

A first approach ${ }^{5}$ modeling the evolution of the ablator properties during the solid-to-plasma transition has recently been developed. The developed model includes electron dynamic upon the laser radiation, and a two-temperature model

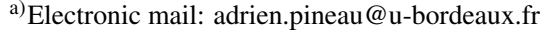

to describe the electron energy transfer to the lattice-ion system. One of the main conclusions is that the solid-to-plasma transition takes place over a timescale of a hundred of picoseconds, which is not small enough to be neglected in comparison to typical ICF laser pulses, of the order of a few nanoseconds. Among the various parameters involved in this model, the electron collision frequency appears to be one of the most important since it drives the laser absorption efficiency and energy transfer from electrons to the lattice-ion system. Within this first approach, a generic description of the electron collision frequency considering both solid and plasma state has been proposed. Electron-phonon collisions are considered in solid state and assumed to be proportional to the temperature of the lattice-ion system. Electron-ion collisions in plasma are modeled with the usual Spitzer's law. These two asymptotic regimes are then linked by the help of the mean free path. Although it led to some first conclusions, it has to be improved for more accurate ICF simulations, especially accounting for the ablator properties under consideration.

The goal of this work is to present an electron collision frequency model in polystyrene including its transformation toward a dense plasma. First of all, it is assumed that electronelectron collisions, which main influence is to make isotropic the electron distribution, can be neglected. This assumption is supported by the fact that the isotropisation takes place over $\sim 1$ ps $^{11}$ which is much shorter than the timescale of solidto-plasma transition $(\sim 100 \mathrm{ps}) \sqrt{5}$. A four-step scenario of the chemical fragmentation of the ablator with the lattice-ion temperature increase is developed (Sec. III). For each state, an accurate expression of the electron collision frequency is derived (Sec. III). Electron collisions with both acoustic and optical phonons are used for solid state. For the plasma state, standard electron-ion collision frequency is adapted for dense plasmas to account for screening effects. Collisions between electrons and neutrals are considered in states between solid and fully 
ionized plasma. An effective collision frequency valid over the whole temperature range reached in ICF experiments is finally presented in Section IV and used to calculate the thermal conductivity evolution with respect to the temperature of the ablator. A good agreement is obtained with thermal conductivity evolution deduced by ab initio calculations. Conclusions and outlooks are drawn in Section $\mathrm{V}$

\section{SCENARIO OF POLYSTYRENE FRAGMENTATION}

This section is devoted to the establishment of a scenario of polystyrene fragmentation in terms of the lattice-ion temperature. Polystyrene is a polymer molecule composed of carbon and hydrogen which the chemical formula is $\left(\mathrm{C}_{8} \mathrm{H}_{8}\right)_{n}$ with $\mathrm{C}_{8} \mathrm{H}_{8}$ the repeat unit and $\mathrm{n}$ their number. Each molecule of polystyrene consists of the repetition of several hundreds of this repeat unit. The structure of polystyrene is given in Fig 1 .

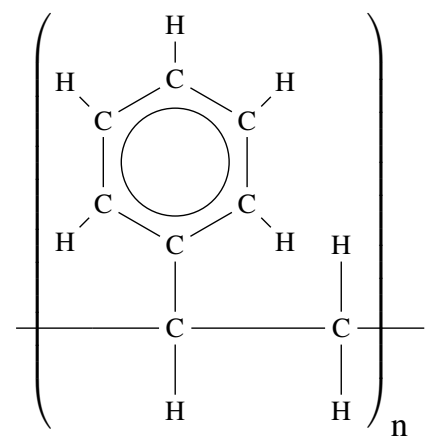

FIG. 1. Chemical structure of polystyrene. The circle indicates that the six $\mathrm{C}-\mathrm{C}$ bonds in the phenyl group are equivalent.

The proposed scenario of polystyrene fragmentation is given in Fig. 2. It presents the successive dissociation reactions of the different species occurring during the polystyrene heating. It has been obtained following an estimation of the chemical bond energies. This is a four-step scenario of fragmentation corresponding to three dissociations that are assumed instantaneous ( $\sim$ ps) compared to the timescale of the solid-to-plasma transition. In addition, the solid-to-plasma transition is assumed to take place over a timescale shorter than the hydrodynamical timescale ${ }^{5}$. This allows to consider the fragmentation as an isochoric process.

At low temperature, the polystyrene is in a solid state. When the lattice temperature is about the melting point, it transforms into a liquid state, but the internal structure of the polystyrene molecules is not modified, polystyrene molecules do not break. As they are made of several hundreds of repeat units, it is assumed that it is still possible to define a local periodicity in the liquid state. Phonons still exist and it is assumed collisions are between electrons and phonons when polystyrene is in this state. Therefore, from a collisional point of view, there is no difference between polystyrene in solid and liquid state.

When the so-called ceiling temperature $T_{c}$ is reached, the depolymerization process (inverse process of polymerization) takes place and the polymer dissociates into monomers. For polystyrene, $T_{c}=58 \mathrm{meV}(670 \mathrm{~K})^{12}$ and becomes styrene $13-16$. The periodicity disappears and phonons are removed. Below $58 \mathrm{meV}$, matter is in the solid state and collisions are between electrons and phonons. Above $58 \mathrm{meV}$, matter is composed of atoms and molecules that will be gradually ionized as the temperature increases. In this regime, electrons collide with neutrals and ions.

When the temperature keeps increasing, styrene dissociates into smaller molecules that dissociate themselves into even smaller molecules up to a fully dissociated state composed only of partially ionized atoms. Each dissociation step is defined by a dissociation temperature. Regarding styrene, the main channel of dissociation gives one molecule of benzene and one molecule of acetylene ${ }^{17}$. This dissociation is expected in regards to the structure of the styrene (see Fig. 2). It is basically composed of a phenyl group and an alkyl group. The phenyl group is difficult to break due to its structure stability. Electrons involved in the carbon-carbon bonds are delocalized all over the cycle and the six bonds are equivalent with an energy between $\mathrm{C}-\mathrm{C}$ and $\mathrm{C}=\mathrm{C}$ bond energies. The alkyl group is composed of one $\mathrm{C}-\mathrm{C}$ bond and one $\mathrm{C}=\mathrm{C}$ bond. Due to a more important atomic orbital overlap in $\mathrm{C}=\mathrm{C}$ than in $\mathrm{C}-\mathrm{C}$, it results the $\mathrm{C}=\mathrm{C}$ bond is stronger than the $\mathrm{C}-\mathrm{C}$ bond. Thus, the $\mathrm{C}-\mathrm{C}$ alkyl bond appears as the weakest and then first breaks. Measurements of styrene fragmentation induced by $308 \mathrm{~nm}$ and $355 \mathrm{~nm}$ lasers showed that the dissociation takes place at $\frac{18}{18} 2.32 \pm 0.1 \mathrm{eV}$ and ${ }^{19} 2.43 \pm 0.05 \mathrm{eV}$ respectively. Therefore, it is assumed that the styrene dissociation happens between $2.32 \mathrm{eV}$ and $2.43 \mathrm{eV}$. Between these two temperatures, both styrene and the benzene-acetylene mixture are considered.

Benzene and acetylene fragmentations should be then considered. Differently from the acetylene fragmentation which products can be easily identified due to its simple structure, the benzene fragmentation is more complicated to determine because of its stability. However, it is possible to assume the only bonds present in the fragments of benzene are $\mathrm{C}-\mathrm{C}$, $\mathrm{C}=\mathrm{C}, \mathrm{C} \equiv \mathrm{C}$ and $\mathrm{C}-\mathrm{H}$. In addition, it is assumed that the aromatic carbon-carbon bond energy are between $\mathrm{C}-\mathrm{C}$ and $\mathrm{C}=\mathrm{C}$ bond energy. Table $\mathrm{I}$ proposes an overview of the different bond energies that can be found in the literature to our knowledge. It is observed that most of bond energies are between 4 and $8 \mathrm{eV}$. Therefore, a lot of molecules and radicals can appear and disappear on this small temperature range (in regards to the solid-plasma transition temperature range). In order not to deal with this very complex mixture evolving very fast, it is set that benzene and acetylene molecules dissociate directly into a carbon-hydrogen mixture between 4 and $8 \mathrm{eV}$. This scenario is supported by the fact that the target remains only a few picoseconds in this temperature range ${ }^{5}$. As for styrene dissociation, between 4 and $8 \mathrm{eV}$ both the benzeneacetylene mixture and the carbon-hydrogen mixture are considered.

A summary of the various species that are considered in terms of the lattice-ion temperature $T_{i l}$ is given in Fig. 3 .

Considering polystyrene at standard density ${ }^{27} \rho_{P S}=$ 


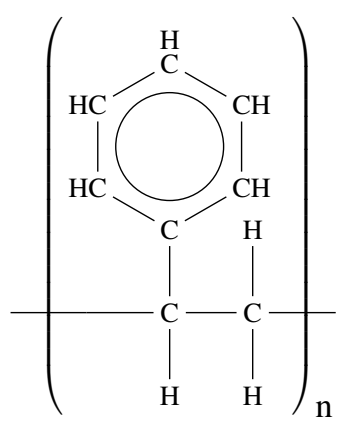

Polystyrene

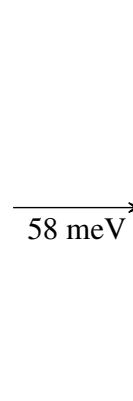

Styrene

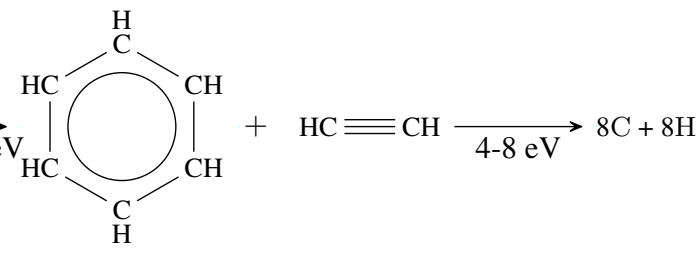

Benzene

Acetylene

FIG. 2. Polystyrene fragmentation model. Only the species are represented, not the matter state (neutral or ionized). The names of the molecules are written underneath.

\begin{tabular}{|c|c|c|}
\hline Chemical bond & Energy bond (eV) & References \\
\hline \multirow[t]{2}{*}{$\mathrm{C}-\mathrm{C}$} & 3.59 & $20]$ \\
\hline & 3.64 & 21 \\
\hline \multirow[t]{2}{*}{$\mathrm{C}=\mathrm{C}$} & 6.35 & 20 \\
\hline & 7.57 & 21 \\
\hline \multirow[t]{2}{*}{$\mathrm{C} \equiv \mathrm{C}$} & 8.70 & 20 \\
\hline & 10.04 & 21 \\
\hline \multirow[t]{2}{*}{$\mathrm{C}-\mathrm{H}$} & 4.32 & 20 \\
\hline & 4.26 & 21 \\
\hline $\mathrm{C}_{6} \mathrm{H}_{5}-\mathrm{H}$ & 4.85 & 22 \\
\hline \multirow[t]{5}{*}{$\mathrm{HCC}-\mathrm{H}$} & $5.65(0 \mathrm{~K}) ; 5.71(298 \mathrm{~K})$ & 23 \\
\hline & 5.73 & 22 \\
\hline & 5.73 & 24 \\
\hline & $5.44-5.68$ & 25 \\
\hline & 5.42 & 26 \\
\hline \multirow[t]{4}{*}{$\mathrm{HC} \equiv \mathrm{CH}$} & $9.84(0 \mathrm{~K}) ; 9.92(298 \mathrm{~K})$ & 23 \\
\hline & 9.80 & 22 \\
\hline & 9.92 & 24 \\
\hline & 9.93 & 26 \\
\hline \multirow[t]{3}{*}{$\mathrm{CC}-\mathrm{H}$ (radical) } & $5.00(0 \mathrm{~K}) ; 5.07(298 \mathrm{~K})$ & 23 \\
\hline & 4.81 & 22 \\
\hline & 5.02 & 24 \\
\hline \multirow[t]{3}{*}{$\mathrm{C} \equiv \mathrm{CH}$ (radical) } & $7.63(0 \mathrm{~K}) ; 7.68(298 \mathrm{~K})$ & 23 \\
\hline & 7.67 & 22 \\
\hline & 7.66 & 24 \\
\hline \multirow[t]{2}{*}{$\mathrm{C} \equiv \mathrm{C}$ (radical) } & 6.06 & 23 \\
\hline & 6.12 & [24] \\
\hline
\end{tabular}

TABLE I. Energies of the bonds involved in the benzene and acetylene fragments and subfragments.

\begin{tabular}{l|c|c|c|c|c} 
PS & S & S+BA & BA & BA+CH & CH \\
\hline
\end{tabular}

FIG. 3. Species considered in terms of the lattice-ion temperature. PS corresponds to polystyrene, $S$ to styrene, BA to the benzeneacetylene mixture and $\mathrm{CH}$ to the carbon-hydrogen mixture.
$1.1 \mathrm{~g} . \mathrm{cm}^{-3}$ leads to a total density of styrene particles (neutrals and ions) $n_{S}=6.31 \times 10^{21} \mathrm{~cm}^{-3}$ between $58 \mathrm{meV}$ and $2.32 \mathrm{eV}$. Between $2.43 \mathrm{eV}$ and $4 \mathrm{eV}$, the total particle density is $n_{B A}=n_{B}+n_{A}=2 n_{S}=1.27 \times 10^{22} \mathrm{~cm}^{-3}$. For temperatures higher than $8 \mathrm{eV}$, the dissociation of benzene and acetylene gives 8 carbon atoms and 8 hydrogen atoms. In that case, the total particle density is $n_{C H}=n_{C}+n_{H}=16 n_{S}=1.02 \times 10^{23}$ $\mathrm{cm}^{-3}$ with $n_{C}$ and $n_{H}$ the density of carbon and hydrogen respectively. In the transition zones, i.e. between 2.32 and $2.43 \mathrm{eV}$, and 4 and $8 \mathrm{eV}$, densities are obtained by linear interpolation.

\section{ELECTRON COLLISIONS}

It was established that two regimes can be exhibited in terms of the lattice-ion temperature. If it is smaller than 58 $\mathrm{meV}$, collisions are between electrons and phonons. If it is higher, electrons collisions are with both neutrals and ions.

\section{A. Electron-phonon collisions}

Polystyrene is a dielectric material which is assumed to be ideal (no impurities nor defects) implying that only electron-phonon collisions take place. As the unit cell of the polystyrene is composed of several atoms, both acoustic and optical phonons have to be taken into account. The collision between electrons and phonons results in the absorption or emission of a phonon by an electron. The electron acoustic phonon (EAP) collision frequency and the electron optical phonon (EOP) collision frequency are given by Eqs. (1) and (2) respectively. The derivation of these collision frequencies is presented in Appendix A.

The (EAP) collision frequency is given by 28 :

$$
v_{A}(\vec{k})=\frac{\mathscr{U}^{2} k_{B} T_{i l} m^{*}}{2 \pi \rho c_{s}^{2} \hbar^{3} k}\left[k^{2}+\left(\frac{m^{*} c_{s}}{\hbar}\right)^{2}\right]
$$

with $k_{B}$ the Boltzmann constant, $\hbar=h / 2 \pi$ the reduced Planck constant, the lattice-ion temperature $T_{i l}$, the material density $\rho$, the sound speed $c_{s}$ and $\mathscr{U}$ the deformation potential. 

by

Electron optical phonon (EOP) collision frequency is given

$$
\begin{array}{r}
v_{L O}(\vec{k})=\frac{e^{2} m^{*} \omega_{L O}}{\hbar^{2} k}\left(\frac{1}{\varepsilon_{r}^{\infty}}-\frac{1}{\varepsilon_{r}^{0}}\right) \times \\
{\left[g_{L O} \ln \left(\frac{\sqrt{1+2 m^{*} \omega_{L O} / \hbar k^{2}}+1}{\sqrt{1+2 m^{*} \omega_{L O} / \hbar k^{2}}-1}\right)\right.} \\
\left.+\left(g_{L O}+1\right) \ln \left(\frac{1+\sqrt{1-2 m^{*} \omega_{L O} / \hbar k^{2}}}{1-\sqrt{1-2 m^{*} \omega_{L O} / \hbar k^{2}}}\right)\right]
\end{array}
$$

The first and second terms in the brackets correspond to the absorption and emission of an optical phonon, respectively. It is expressed in terms of the elementary charge $e$, the optical phonon energy $\hbar \omega_{L O}$, the dielectric constant $\varepsilon_{r}^{0}$ and $\varepsilon_{r}^{\infty}$ at low and high frequency, respectively, and the phonon distribution function $g_{L O}=\left[\exp \left(\hbar \omega_{L O} / k_{B} T_{i l}\right)-1\right]^{-1}$. For polystyrene, the dielectric constants are ${ }^{\sqrt{12}} \varepsilon_{r}^{0}=2.5$ and ${ }^{29} \varepsilon_{r}^{\infty}=1.01$.

In order to get a macroscopic description for ICF purpose, these frequencies are averaged over the Brillouin zone weighted by the electron distribution function $f(\vec{k})$ and the density of states $g(\vec{k})$ :

$$
\bar{v}=\frac{\int_{k_{\min }}^{k_{B Z}} v(\vec{k}) g(\vec{k}) f(\vec{k}) \mathrm{d} \vec{k}}{\int_{k_{\min }}^{k_{B Z}} g(\vec{k}) f(\vec{k}) \mathrm{d} \vec{k}}
$$

For acoustic phonons, the different assumptions allowing to obtain Eq. (1) have led not to make differences between electron collisions by phonon emission or by phonon absorption. Therefore, one has $k_{\min }=m^{*} c_{s} / \hbar$ for EAP collision frequency because an electron cannot emit a phonon if its momentum is smaller than the phonon momentum. For EOP collision frequency, one has $k_{\min }=0$ for absorption because an electron can absorb a phonon regardless of its momentum, and $k_{\min }=k_{L O}$ for emission in the same way as for acoustic phonons. Note that in practice Eq. (3) is split into two parts in order to calculate the emission part and the absorption part separately. The density of states $g(\vec{k})$ in Eq. (3) is taken in the free electron gas approximation $g(\vec{k})=2 \mathrm{~V} / 8 \pi^{3}$ and the electron distribution function is given by the Fermi-Dirac distribution function $f(\vec{k})=\left[\exp \left((E(\vec{k})-\mu) / k_{B} T_{e}\right)+1\right]^{-1}$ where the energy of the bottom of the conduction band has been set to zero. An approximation of the chemical potential $\mu$ is derived in Appendix $C$.

Two optical phonons are considered for polystyrene with $\hbar \omega_{L O, 1}=33.53 \mathrm{meV}$ and $\hbar \omega_{L O, 2}=88.73 \mathrm{meV}$. The derivation of the relation dispersion leading to the phonon number and the optical phonon energies is given in Appendix B Finally, the total electron collision is given by the Mathieusen rule ${ }^{30}$ :

$$
v_{e-p h}=\bar{v}_{A}+\bar{v}_{L O, 1}+\bar{v}_{L O, 2}
$$

Figure 4 presents the total electron-phonon collision frequency given by Eq. (4) as a function of $T_{e}$ and $T_{i l}$, for $T_{i l} \leq 58 \mathrm{meV}$ and for $T_{e}>T_{i l}$ because $T_{e}<T_{i l}$ is usually not observed in ICF experiments. It is set $n_{e}=10^{10} \mathrm{~cm}^{-3}$ corresponding to the number of electrons that are present in

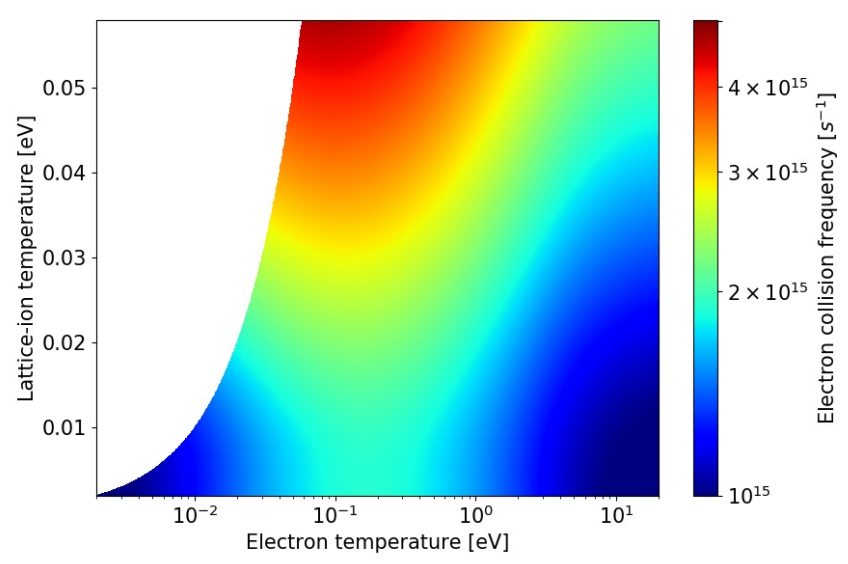

FIG. 4. Electron-phonon collision frequency as a function of the electron and lattice-ion temperatures assuming a free electron density $n_{e}=10^{10} \mathrm{~cm}^{-3}$. It is plotted for $T_{e}>T_{i l}$ which account for realistic physical conditions.

the conduction band of dielectrics ${ }^{31}$. Note that the electronphonon collision frequency depends on the electron density due to the chemical potential. Within ICF conditions, values of both electron temperature and density are such tha $t^{5}$ the chemical potential is defined by Eq. C6. Thus, the chemical potential exhibits a logarithmic dependence and electron density variations will slightly modify the collision frequency.

The increase in the collision frequency with the latticeion temperature is due to phonon population increasing. The higher the temperature $T_{i l}$, the larger the phonon density, and the larger the number of collisions. The evolution of the collision frequency as a function of $T_{e}$ is similar to the behavior of the collision frequency as a function of the electron energy $E(\vec{k})$, especially the one related to the emission of an optical phonon by an electron,that is defined by Eq. (2). Note that Eq. (2) can be written with respect to the electron energy by using $E(\vec{k})=\hbar^{2} k^{2} / 2 m^{*}$. This collision frequency first increases with the electron energy up to a maximum where the electron energy becomes of the order of magnitude of the phonon energy, and decreases for higher electron energies. Such a behavior is standard for electron optical phonon collision frequency 32 . In addition, values of the total collision frequency lie in the range of other dielectrics, as silicon dioxide ${ }^{32}$.

\section{B. Electron collisions in the dense plasma state}

In the plasma state, electrons can collide with ions and neutrals. These collisions depending on the particles (electrons, ions and neutrals) densities and the Coulomb logarithm, the latter are first evaluated before presenting the collision frequencies. 


\section{Ionization}

This subsection is devoted to the presentation of the models used to describe ionization of styrene, benzene, acetylene, carbon and hydrogen. Thermal ionization models are considered.

Ionization of styrene, benzene and acetylene is described by the Saha equation. Because styrene exists for temperatures smaller than $2.43 \mathrm{eV}$ (see Fig. 3), only the first ionization is considered. In the same way, benzene and acetylene exist for temperatures smaller than $8 \mathrm{eV}$ and only the first ionization state are considered. Ionization energies for styrene, benzene and acetylene are $\mathrm{e}^{17 / 33} 8.46 \mathrm{eV}, 9.24 \mathrm{eV}$ and $11.42 \mathrm{eV}$, respectively. Ionization of the $\mathrm{CH}$ mixture is obtained by ab initio calculations. As it exists starting from $4 \mathrm{eV}$, all of the ionization states are considered which ionization energies range from $11.26 \mathrm{eV}$ to $490 \mathrm{eV}$. Saha equations and the model associated to ab initio calculations are detailed in Appendix D.

Figure 5 (a) shows the averaged ionization state $\langle Z>$ as a function of temperature. Assuming that the plasma behavior, i.e. when density of charged particles in the medium becomes large enough so that its properties are modified, appears from an ionization degree ${ }^{34} \alpha=n_{i} / n_{T} \approx 10^{-3}$, it can be deduced from Fig. 5(a) that the plasma behavior starts from around $650 \mathrm{meV}$. Note that for low temperatures, $n_{e} \approx n_{i}$ and $\alpha \approx\langle Z\rangle$. Therefore, between $58 \mathrm{meV}$ and $650 \mathrm{meV}$, styrene can be considered as a dense neutral gas.

Figure 5(b) displays the density of electrons, neutrals and ions between $58 \mathrm{meV}$ and $1 \mathrm{keV}$ for $T_{e}=T_{i l}$. It appears that neutrals are mainly present for temperatures smaller than around $4 \mathrm{eV}$. A drop in the neutral density is observed beyond this temperature because it has been defined as $n_{n}=n_{T}-n_{i}$ where $n_{i}=\min \left(n_{e}, n_{T}\right)$ for the $\mathrm{CH}$ mixture. Because the ionization model of $\mathrm{CH}$ gives only the electron density, it is impossible to access to the density of each ion population. Therefore, it is assumed that when electron density is higher than the total density, it means that all of the atoms are ionized so the density of neutral particles drops to zero. This may result in an overestimation of the ion density and an underestimation of the neutral density for temperature around $10 \mathrm{eV}$.

It is possible to deduce that electron-neutral collisions are the dominant process for temperatures up to $4 \mathrm{eV}$, there is a competition between electron-neutral and electron-ion for temperatures between 4 and $8 \mathrm{eV}$, and beyond, electron-ion collisions are the dominant process.

\section{Coulomb logarithm}

Usually, the Coulomb logarithm $\ln \Lambda$ appearing in the electron-ion collision frequency is obtained assuming particles interacting in a Coulomb potential, i.e. without screening effects. In such a potential, the Coulomb logarithm reads in the well-known form :

$$
(\ln \Lambda)_{C}=\frac{1}{2} \ln \left(1+\frac{b_{\max }^{2}}{b_{\min }^{2}}\right)
$$
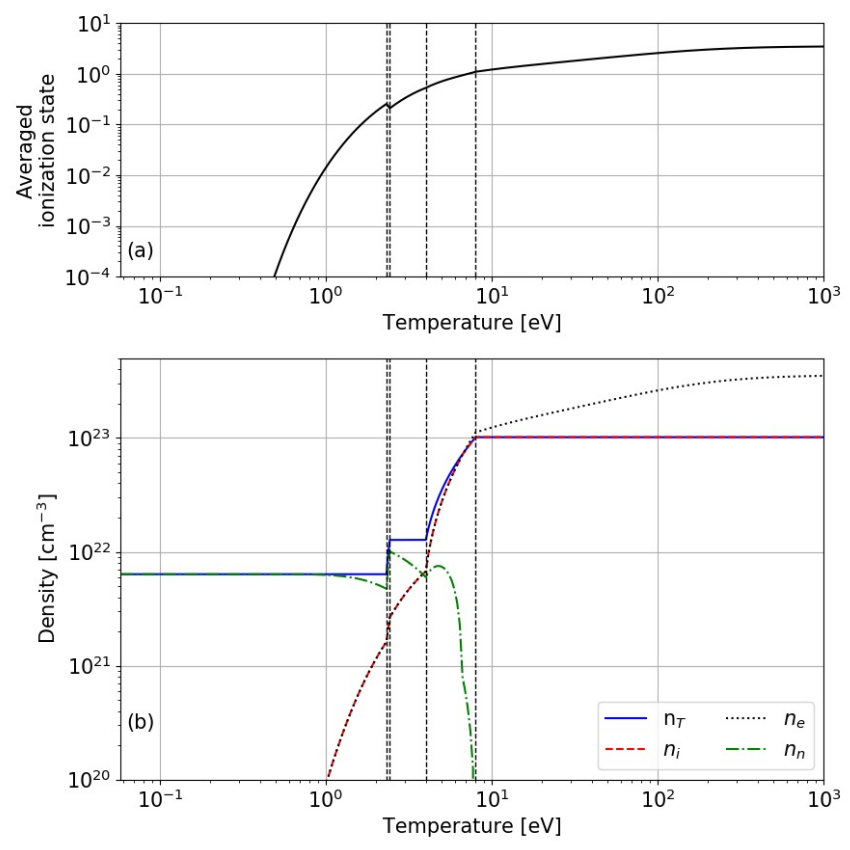

FIG. 5. (a) Averaged ionization state $\langle Z\rangle$ as a function of the temperature. (b) Density of species as a function of the temperature. The dotted curve represents the electron density $n_{e}$, the dashed curve the total ion density $n_{i}$, the dash-dot curve the total neutral density $n_{n}$ and the solid curve the total particles density $n_{T}=n_{n}+n_{i}$. Vertical dashed lines indicate dissociation temperatures at 2.32, 2.43, 4 and 8 eV. Both (a) and (b) are plotted for $T_{e}=T_{i l}$.

with $b_{\text {min }}$ and $b_{\text {max }}$ the lower and upper cut-off lengths respectively. In dense plasmas, screening effects have to be taken into account and the previous definition is no longer valid. Based on an evaluation of the stopping power, a definition of the Coulomb logarithm available for any potential $U(r)$ has been proposed 35 :

$$
\ln \Lambda=\frac{1}{b_{\min }^{2}} \int_{0}^{b_{\max }} b \sin ^{2}\left(\frac{\theta}{2}\right) \mathrm{d} b
$$

with $b$ the impact parameter. The scattering angle $\theta$ of the electron is defined by

$$
\theta=\pi-2 b \int_{r_{0}}^{+\infty} \frac{1}{r^{2}}\left(1-\frac{2 U(r)}{k_{B} T_{e}}-\frac{b^{2}}{r^{2}}\right)^{-1 / 2} \mathrm{~d} r
$$

where $r_{0}$ is the distance of closest approach and satisfies the relation $1-2 U\left(r_{0}\right) / k_{B} T_{e}-b^{2} / r_{0}^{2}=0$. Interactions between ions of charge $Z e$ and electrons are modeled by the DebyeHückel potential $U_{D H}(r)$ which is the generalization of the Coulomb potential including screening effects ${ }^{37}$ :

$$
U_{D H}(r)=\frac{Z e^{2}}{r} \exp \left(-\frac{r}{\lambda_{D H}}\right)
$$


The Debye-Hückel length $\lambda_{D H}$ characterizing the screening effects is written in a two-temperature mode ${ }^{38}$ :

$$
\frac{1}{\lambda_{D H}^{2}}=\frac{4 \pi n_{e} e^{2}}{k_{B} \sqrt{T_{e}^{2}+T_{F}^{2}}}+\frac{4 \pi n_{i} Z^{2} e^{2}}{k_{B} T_{i l}}
$$

where the Fermi temperature $T_{F}$ allows to take into account the degeneracy correction appearing in a dense plasma within a good approximation 39 . By using Eq. (8), the distance of closest approach for $b=0$ is given by :

$$
r_{\perp}=\lambda_{D H} \mathrm{Wp}\left(\frac{2 Z e^{2}}{\lambda_{D H} k_{B} T_{e}}\right)
$$

where $\mathrm{Wp}$ is the principal branch of the Lambert Wfunction ${ }^{40}$. Finally, the parameters $b_{\min }$ and $b_{\max }$ in Eq. (6) are defined as :

$$
b_{\text {min }}=\frac{1}{2} \max \left[r_{\perp}, \lambda_{B}\right] \quad ; \quad b_{\max }=\sqrt{\lambda_{D H}^{2}+a_{i}^{2}}
$$

with $\lambda_{B}=\hbar / \sqrt{m_{e} k_{B} T_{e}}$ the De Broglie length $\frac{41}{4}$ ensuring to respect the Heisenberg uncertainty principle ${ }^{42}$ and $a_{i}=$ $\left(3 / 4 \pi n_{i}\right)^{1 / 3}$ the ion sphere radius allowing to take into account the effects of a high density plasma 43 . Note that the integration in Eq. (6) is done up to $b=b_{\max }$ and not $b \rightarrow+\infty$. It has been assumed that $U(r)=0$ for $r>\lambda_{D}$ leading to $\theta=0$. This ensures the convergence of the Coulomb logarithm while having a screened potential inside the Debye sphere and without double counting screening effects.

By modeling a $\mathrm{CH}$ plasma over a wide range of temperatures and densities occurring in ICF with ab initio calculations based on quantum molecular dynamics, a fitted formula for a generalized Coulomb logarithm $(\ln \Lambda)_{Q M D}$ has been obtained ${ }^{44}$. These ab initio calculations were performed using quantum molecular dynamics methods where the electronic potential is evaluated by the density functional theory. A CH plasma was modeled over a wide range of temperatures and densities relevant of ICF applications. The Coulomb logarithm was deduced from the thermal conductivity calculated in terms of the Onsager coefficients using the Kubo-Greenwood formalism. This allows to examine the validity of the previous two Coulomb logarithm evaluations designed to be introduced in models of laser-matter interaction.

Figure 6compares the usual Coulomb logarithm $(\ln \Lambda)_{C}$ defined by Eq. (5), the Coulomb logarithm $(\ln \Lambda)_{D H}$ defined by Eq. (6)-(7) using the Debye-Hückel potential, and the QMD Coulomb logarithm $(\ln \Lambda)_{Q M D}$ for $T_{e}=T_{i l}$. The latter is plotted only for $T>8 \mathrm{eV}$ since it has only been developed for a $\mathrm{CH}$ mixture. It is reminded a pure $\mathrm{CH}$ mixture is considered in this work only for $T>8 \mathrm{eV}$ (see Fig. 3).

It appears that $(\ln \Lambda)_{C}$ cannot be used for the range of temperature and density of ICF. Despite a reasonable order of magnitude for temperatures higher than $100 \mathrm{eV}$, it underestimates by a factor from 2 to 4 for temperatures between 8 and $100 \mathrm{eV}$. Moreover, it shows a non-physical behavior for temperatures smaller than $8 \mathrm{eV}$ as it increases when the temperature decreases. This is due to the cut-off lengths. For $(\ln \Lambda)_{C}$, they are defined by Eq. $\left[11\right.$, as for $(\ln \Lambda)_{D H}$, except that $r_{\perp}=$

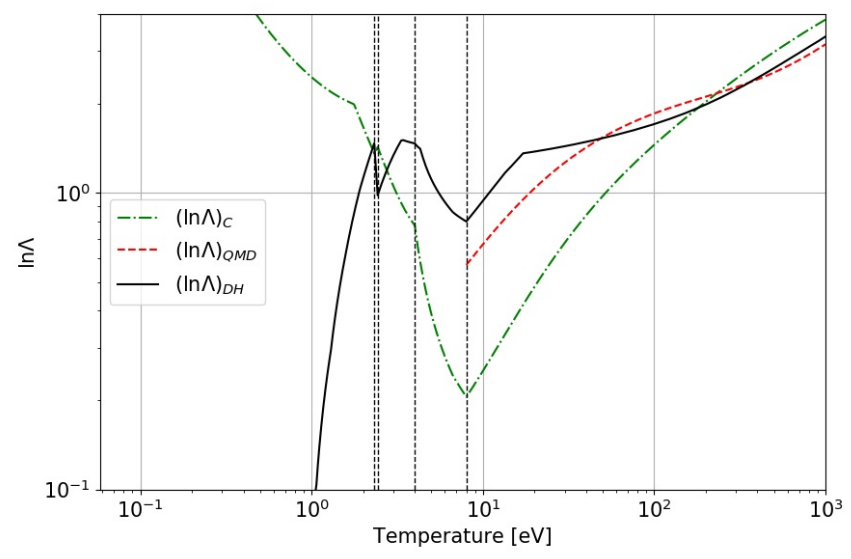

FIG. 6. Coulomb logarithm for various models as a function of the temperature. Calculations have been done with $T_{e}=T_{i l}$. It is reminded vertical dashed lines indicate dissociation temperatures at 2.32, 2.43, 4 and $8 \mathrm{eV}$.

$2 Z e^{2} / k_{B} T_{e}$. When $T_{e}$ becomes very small, one has $b_{\min } \approx \lambda_{B}$ and $b_{\max } \approx \lambda_{D H}$. Thus, $b_{\max }^{2} / b_{\min }^{2} \approx m_{e} k_{B}^{2} T_{e}^{2} / \pi \hbar^{2} e^{2} n_{e}$ using the fact that $\lambda_{D H} \approx k_{B} T_{e} / 4 \pi n_{e} e^{2}$ for small electron temperatures. Because a small variation of temperature leads to large variation of $n_{e}$ (see Fig. 55, the ratio $b_{\max }^{2} / b_{\min }^{2}$ is driven by the behavior of $n_{e}$. So $b_{\text {max }}^{2} / b_{\text {min }}^{2} \rightarrow+\infty$ and $(\ln \Lambda)_{C} \rightarrow+\infty$ when the temperature becomes very small.

Relatively good agreements are observed between $(\ln \Lambda)_{D H}$ and $(\ln \Lambda)_{Q M D}$. Despite differences of $40 \%$ can be observed for temperatures around $10 \mathrm{eV}$, they are quite similar for temperatures higher than $40 \mathrm{eV}$. For temperatures smaller than about $2 \mathrm{eV}$, the definition of $(\ln \Lambda)_{D H}$ proposed in this work goes wrong as it leads to $(\ln \Lambda)_{D H} \ll 1$. Thus, in that case, we impose $(\ln \Lambda)_{D H}=1$. However, the consequences of not correctly evaluating $\ln \Lambda$ in this domain are relatively not important because the dominant processes are collisions between electrons and neutrals.

It can be noticed that the slope-changing points at $2 \mathrm{eV}$ for $\ln \Lambda_{C}$ and at 3 and $20 \mathrm{eV}$ for $\ln \Lambda_{D H}$ correspond to the temperatures where the switch between $\lambda_{B}$ and $r_{0}$ takes place in Eq. [11.).

\section{Collision frequencies}

For electron-neutral collisions, a simple model based on the classical diffusion cross section $\sigma$ is used ${ }^{41}$ :

$$
v_{e n}=n_{n} \sigma \sqrt{\frac{k_{B} T_{e}}{m_{e}}}
$$

with $n_{n}$ the density of neutral. The diffusion cross section is expressed by assuming neutrals as hard spheres and neglecting the deformation of the electronic cloud during the collision with an electron. Then $\frac{45}{,} \sigma=\pi a^{2}$ with $a$ the characteristic size of the neutral ranging from several tens to several hundreds of ångströms depending on if atoms or molecules 
are considered. Polarization effects due to the interaction between an electron and the electric dipole moment of neutral particles are indeed neglected in our expression of electronneutral collisions. This assumption is supported by the following considerations. Styrene, benzene and acetylene are nonpolar molecules, i.e. the total permanent dipole moment is equal to zero. Because permanent dipole moments of each $\mathrm{C}-\mathrm{H}$ bond cancel each other out due to the molecule symmetry. In addition, models based on induced polarization are relevant for temperatures higher than several $\mathrm{eV}$ for which the plasma is almost fully ionized in our model and electronneutral collisions are negligible. Thus the polarization influence for electron scattering onto neutrals is expected to be negligible within the present conditions.

For laser intensities in ICF applications, the electron quiver velocity is generally lower than the electron thermal velocity. Electron-ion collisions can thus be described in the classical regime $46-50$. Since the solid-to-plasma transition takes place on a timescale smaller than hydrodynamic timescale ${ }^{5}$, the plasma density remains close to the solid density. The Spitzer's model is no longer valid and the Lee and More model is used 38 .

$$
v_{e i}=\frac{2 \sqrt{2} \pi}{3} \frac{Z^{2} e^{4} n_{i}}{\sqrt{m_{e}}\left(k_{B} T_{e}\right)^{3 / 2}} \frac{(\ln \Lambda)_{D H}}{\left(1+\exp \left(-\mu / k_{B} T_{e}\right)\right) F_{1 / 2}}
$$

where $F_{1 / 2}$ is the complete Fermi-Dirac integral:

$$
F_{1 / 2}\left(\frac{\mu}{k_{B} T_{e}}\right)=\int_{0}^{+\infty} \frac{\sqrt{t}}{1+\exp \left(t-\mu / k_{B} T_{e}\right)} \mathrm{d} t
$$

and $\mu$ is defined by Eq. (C6)-( $(\ln \Lambda)_{D H}$ defined by Eq. (6)-(8) is used to take into account the screening effects.

Figure 7 shows the total electron collision frequency $v_{e i}+$ $v_{e n}$ as a function of electron and lattice-ion temperatures between $58 \mathrm{meV}$ and $1 \mathrm{keV}$. As for the electron-phonon collision frequency, it is plotted for $T_{e}>T_{i l}$ since it is usually not observed $T_{e}<T_{i l}$ in ICF experiments.

The general behavior of the electron collision frequency is relatively standard. It increases with the lattice-ion temperature because when $T_{i l}$ increases, the successive chemical dissociations of the ablator lead to a growing number of atoms and molecules. Concerning its evolution as a function of the electron temperature, it first increases between $58 \mathrm{meV}$ and around a few $\mathrm{eV}$. The electron temperature is not large enough to ionize molecules, so the density of neutrals remains quite constant and the electron collision frequency increases with the electron temperature (see Eq. (12)). For higher electron temperatures, atoms and molecules are ionized and the decrease in the electron collision frequency corresponds to the characteristic behavior of electron-ion collisions, i.e. the electron collision frequency evolves as $T_{e}^{-3 / 2}$ (see Eq. (13)). It appears electron-neutral collisions are the dominant process for electron temperature smaller than a few $\mathrm{eV}$ and beyond, electron-ion collisions are the dominant process.

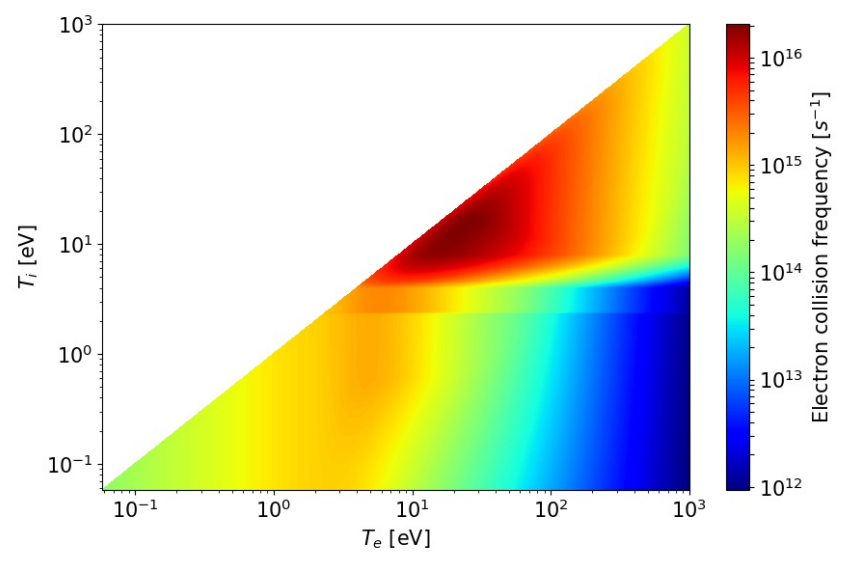

FIG. 7. Total electron collision frequency $v_{e i}+v_{e n}$ in the plasma state as a function of electron and lattice-ion temperatures for $T_{e}>T_{i l}$ which account for realistic physical conditions.

\section{APPLICATIONS OF DERIVED COLLISION FREQUENCIES}

\section{A. Effective electron collision frequency}

A collision frequency $v_{\text {eff }}$ valid over the whole range of temperature occurring in inertial confinement fusion can be deduced from what has been presented previously. If $T_{i l} \leq T_{c}$, with $T_{c}=58 \mathrm{meV}$, the ablator is in the solid state and electrons collide with phonons. Otherwise, it is a dense partially ionized gas and electrons collide with neutrals and ions. Thus $v_{\text {eff }}$ reads :

$$
v_{e f f}=v_{e p h} x_{P S}+\left(v_{e i}+v_{e n}\right) x_{A M}
$$

where $v_{e p h}, v_{e n}$ and $v_{e i}$ are defined by Eq. (4), Eq. (12) and Eq. (13), respectively. Fractions $x_{P S}$ and $x_{A M}$, which are both function of the temperature, represent fractions of polymer and atoms/molecules densities respectively. They are the dominant species for temperatures smaller and higher than $T_{C}$ respectively. These fractions satisfy $x_{P S} \rightarrow 1$ and $x_{A M} \rightarrow 0$ when $T \ll T_{c}, x_{P S} \rightarrow 0$ and $x_{A M} \rightarrow 1$ when $T \gg T_{c}$, and $x_{P S}=x_{A M}=1 / 2$ when $T=T_{c}$. Variations of the species fractions around the transition are described by a second-order polynome. Such a definition allows to have an effective collision frequency that is continuous during the transition from the solid state to the plasma state taking place over a range $\delta T$ around $T_{c}$. This temperature range being much smaller than the one considered to plot the effective collision frequency $\sqrt{51}$ in Fig. 8, this transition appears as a jump.

It can be seen that when the ablator is in the solid state, the collision frequency increases with the temperature. This is due to the excitation of phonon modes. At $T=58 \mathrm{meV}$, a drop by a factor 10 is induced by both phonon removal and small electron temperature in the plasma state. Then, due to the increase of electron density and temperature, the collision frequency increases up to around $10^{16} \mathrm{~s}^{-1}$ for temperatures 


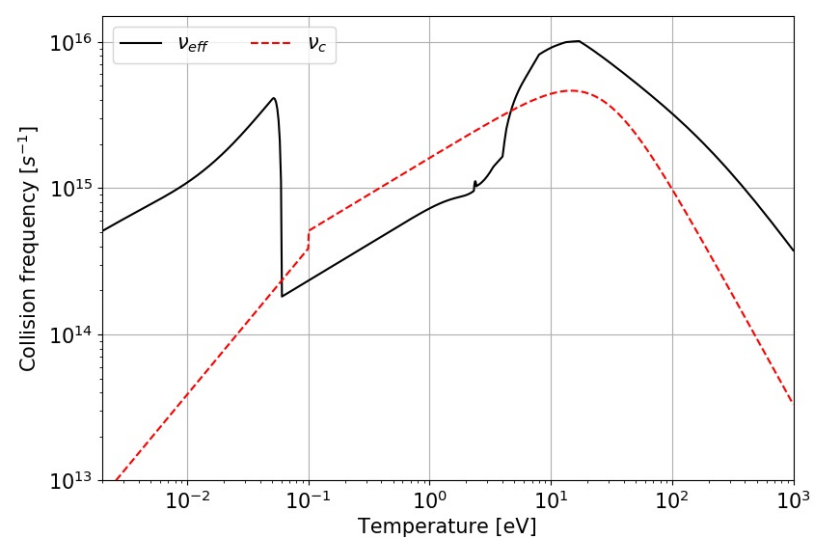

FIG. 8. Effective collision frequency $v_{e f f}$ developed in this work and the generic electron collision frequency $v_{c}$ used in 5 as a function of the temperature assuming $T_{e}=T_{i l}$.

between $15 \mathrm{eV}$ and $20 \mathrm{eV}$. Finally, beyond $20 \mathrm{eV}$, the collision frequency decreases with a typical plasma behavior.

The generic electron collision frequency $v_{c}$ developed in $\frac{5}{5}$ is also presented in Fig. 8. In that previous work, the collision frequency is defined as $v_{c}=v_{0} T_{i l} / T_{0}$ with $v_{0}=10^{14}$ $\mathrm{s}^{-1}$ and $T_{0}=300 \mathrm{~K}$ if $T_{i l}<0.1 \mathrm{eV}$. If $T_{i l}>0.1 \mathrm{eV}, v_{c}^{-1}=$ $v_{m p f}^{-1}+v_{e-i}^{-1}$ which is defined in terms of $v_{m f p}=v_{e} n_{a}^{1 / 3}$ the mean free path with $v_{e}$ and $n_{a}=3 \times 10^{22} \mathrm{~cm}^{-3}$ the electron thermal velocity and the atomic density respectively, and $v_{e-i}=3.10^{-6} Z_{e} T_{e}^{-3 / 2} \ln \Lambda$ the usual Spitzer's electron-ion collision frequency with $T_{e}$ in $\mathrm{eV}$ and assuming $Z \ln \Lambda=10$, and $n_{e}=3 \times 10^{22} \mathrm{~cm}^{-3}$ which corresponds to the order of magnitude of the critical density used in hydro-codes as initial condition. Note that this is a generic approach in the sense that it does not account for material specificities as its chemical fragmentation.

Large differences can be observed between the generic collision frequency and the one of this work in terms of behavior and amplitude depending on the temperature. At low temperatures, this is due to the fact that evaluating the electronphonon collision frequency as $v_{0} T_{i l} / T_{0}$ includes only collisions between electrons and acoustic phonons. Collisions between electrons and optical phonons are neglected. However, it has been showr ${ }^{32}$ that in dielectrics, electron-optical phonon collisions are the dominant processes and electron-acoustic phonon collisions only gives a small correction to the total electron-phonon collision frequency. At high temperatures, the difference by a factor of 10 is explained by the difference by a factor of 10 between electron densities used in the various collision frequencies. As mentioned previously, a large jump around $T_{c}$ seems to appears. In addition to the fact that the energy range of Fig. 8 does not allow to see the continuous transition at $T_{c}$, this jump also appears because the total collision frequency is plotted for $T_{e}=T_{i l}$ which does not represent relevant conditions of ICF. Within ICF conditions, when polystyrene depolymerizes at $0.058 \mathrm{eV}$, electron temperature is about a few $\mathrm{eV}^{\sqrt{5}}$. Thus, having an electron temperature of a few $\mathrm{eV}$ instead of $0.058 \mathrm{eV}$ leads to a decrease of the electronphonon collision (see Fig. 4) and an increase of the electronneutral collision frequency (see Fig. 7), so a reduction of the jump.

Despite such discrepancies, a relatively small difference in the solid-to-plasma transition timescale is expected because it mainly depends on the time required to reach the critical plasma density, the latter being mainly imposed by the photoionization rate $\mathrm{e}^{5}$ that is not considered in this work. To include photoionization processes, it is necessary to model the electron dynamics coupled to a description of the temporal evolution of both electron and ion-lattice temperatures. This is beyond the scope of the present work as done in 5 .

However a significant influence on both optical and thermal matter responses is expected. It appears from Fig. 8 that the effective collision frequency is mostly smaller than the laser pulsation considering a $351 \mathrm{~nm}$ laser. This leads to a conductivity and a laser absorption proportional to the collision frequency. Thus, higher conductivity and laser absorption for smallest (solid state) and highest temperatures (full plasma state) are expected. In the transition zone for temperatures around a few eV, a smaller conductivity is expected. In addition, note that their value is also strongly driven by the free electron density that, in case where the laser interaction is included in the modeling, is driven by photoionization processes. Such a study is out of the scope of the present work and will be adressed in a forthcoming study.

\section{B. Plasma thermal conductivity}

In order to evaluate the thermal response of the ablator, this section is devoted to the evaluation of the thermal conductivity. Here, only the plasma state for temperatures higher than $1 \mathrm{eV}$ is considered so that the electron density is large enough to ensure that the thermal conductivity is mainly due to electron collisions (which are well described in the present modeling). Below $1 \mathrm{eV}$, the ionized degree is very low (see Fig 5, the contribution of neutrals to the thermal conductivity is thus significant, and the present modeling no longer holds in that case.

Starting from the electron collision frequency in the plasma state, it is possible to deduce the thermal conductivity. This is a quantity of interest because it is involved (i) in the heat transport in plasmas, (ii) it is related to the evolution of hydrodynamic instabilities and (iii) it is one of the key parameter for target ablation when the electron density becomes higher than the critical density.

The thermal conductivity $\kappa_{t}$ in a dense plasma can be described by the Lee \& More model 38 . However, electronelectron collisions are neglected in this model leading to an overestimation of the thermal conductivity. To include these collisions, the Lee \& More model is weighted by a function $R(Z)$ that can be defined as $R(Z)=(Z+0.25) /(Z+4.26)$. Thus, the thermal conductivity $\kappa_{t}$ is given by :

$$
\kappa_{t h}=\frac{n_{e} k_{B}\left(k_{B} T_{e}\right)}{m_{e} v_{e f f}} A\left(\frac{\mu}{k_{B} T_{e}}\right) R(Z)
$$


with the function $A$ defined by :

$$
A\left(\frac{\mu}{k_{B} T_{e}}\right)=\frac{20}{9} \frac{F_{4}\left(1-16 F_{3}^{2} / 15 F_{2} F_{4}\right)}{\left[1+\exp \left(-\mu / k_{B} T_{e}\right)\right] F_{1 / 2}^{2}}
$$

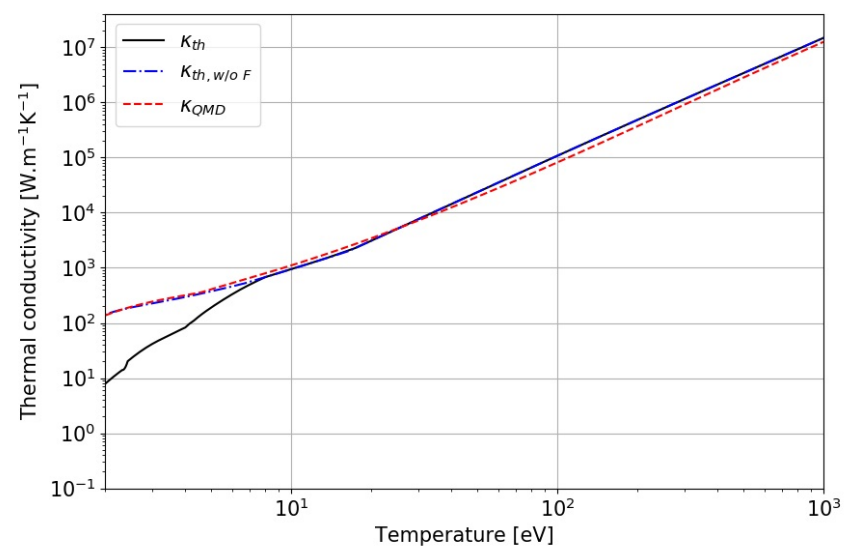

FIG. 9. Analytical $\kappa_{t h}$, analytical $\kappa_{t h, w / o F}$ without the fragmentation processes and ab initio $\kappa_{Q M D}$ thermal conductivities as a function of the temperature with $T_{e}=T_{i l}$.

A good agreement is observed in Fig. 9 for temperatures higher than $8 \mathrm{eV}$. Differences for temperatures smaller than $8 \mathrm{eV}$ come from the fact that the ab initio model considers a $\mathrm{CH}$ mixture for any temperatures and neglects the chemical fragmentation of the ablator below $8 \mathrm{eV}$ whereas this fragmentation is taken into consideration in the present work (see Fig. 3). Below $8 \mathrm{eV}$, the ablator is mainly composed of benzene and acetylene, and below $2.3 \mathrm{eV}$, it is mainly composed of styrene. Thus, the total density is smaller leading to an electron density smaller than the one in ab initio calculations. It results $\kappa_{t h}$ becomes smaller than $\kappa_{Q M D}$ for temperatures below $8 \mathrm{eV}$. This origin of the differences is confirmed by comparing $\kappa_{t h, w / o} F$ to the other two as it corresponds to the thermal conductivity defined by Eq. (16) but where the fragmentation processes are not taken into account and a plasma composed of carbon and hydrogen is considered.

This smaller thermal conductivity will lead to a smaller heat flux and thus a slower heating of the ablator. Because at the same time, the ablator is ionized by the laser pulse, the conduction zone will be smaller when the critical density will be reached. In the end, thermal smoothing occurring in the conduction zone will be less efficient and the laser imprint will be more important as more perturbation modes will be able to be transmitted to the ablation front.

\section{v. CONCLUSION}

Two collision frequencies relevant from inertial confinement fusion conditions have been presented in this work. An electron-phonon collision frequency taking both acoustic and optical phonons into consideration has been derived in order to describe electron collisions when the ablator is in the solid state. Electron collisions when the ablator is in the plasma state are described considering both electron-neutral and electron-ion collisions. For the latter, a modified Coulomb logarithm has been used in order to account for screening effects, showing a good agreement when compared to ab initio calculations. By coupling these two collision frequencies with a four-step fragmentation model for polystyrene ablator, which describes the successive chemical dissociations of polystyrene leading to a carbon-hydrogen mixture, an effective collision frequency valid over the whole range of temperatures reached in inertial confinement fusion experiments has been obtained. This effective collision frequency has been compared to a generic collision frequency used to describe the solid-to-plasma transition of the ablator. Relatively large differences in behavior and amplitude depending on temperature are observed but the timescale of solid-to-plasma transition should not be strongly modified as it mainly depends on the photo-ionization rate. However, larger modifications of thermal and hydrodynamic responses are expected since they are driven by the electron collision frequency due to laser heating. In addition, this effective collision frequency has been used to evaluate the thermal conductivity for temperatures above $1 \mathrm{eV}$. When compared to a thermal conductivity obtained by ab initio calculations, good agreements have been observed for temperatures larger than $10 \mathrm{eV}$. But below $10 \mathrm{eV}$, a smaller conductivity was found, coming from the consideration of the chemical fragmentation of the ablator, which could lead to a more important laser imprint.

Such an accurate description of the collision frequency will be used in future studies dedicated to model the laser-induced solid-to-plasma transition.

\section{ACKNOWLEDGMENTS}

The authors acknowledge Vladimir Tikhonchuk for fruitful discussions about Coulomb logarithm. Patrick Mora is also gratefully acknowledged for his input on the thermal conductivity. This study has been carried out with financial support from the French State, managed by the French National Research Agency (ANR) in the frame of "the Investments for the future" Programme IdEx Bordeaux - LAPHIA (ANR-10IDEX-03-02). CEA is also acknowledged for supporting these studies.

\section{DATA AVAILABILITY STATEMENTS}

The data that support the findings of this study are available from the corresponding author upon reasonable request.

\section{Appendix A: Electron-phonon collision frequency}

Electrons in the conduction band are described under the parabolic band approximation and their energies are given by 
$E(k)=\hbar^{2} k^{2} / 2 m^{*}$, with $m^{*}$ the effective electron mass assumed to be equal to the free electron mass. Electron-phonon collisions are assumed to be limited to the first Brillouin zone as electron energies do not exceed the energy $E\left(k_{B Z}\right)$ associated to the Brillouin zone wave vector $k_{B Z}$. This implies that umklapp processes, corresponding to collisions where the reciprocal lattice vector has to be considered in crystal momentum conservation $[33$, are not considered. Because the number of phonons and their respective energies are calculated in one dimension (see Appendix B), the Brillouin zone wave vector reads $k_{B Z}=2 \pi / a$ with $a=2 d_{C-C}$ the size of the unit cell and ${ }^{54} d_{C-C}=1.53 \AA$ the distance between two atoms of the carbon chain. This leads to $E\left(k_{B Z}\right) \approx 16 \mathrm{eV}$. Note that it has been assumed in Section [I] polystyrene is considered for $T_{i l} \leqslant$ $58 \mathrm{meV}$. In a one-temperature model, $T_{e}=T_{i l}$ and it clearly appears electrons have an energy much smaller than $E\left(k_{B Z}\right)$. But, in the laser-matter interaction framework, electrons are heated first, well before the ion-lattice system. However, even in that case, it has been shown $\sqrt{5}$ that when $T_{i l} \approx 58 \mathrm{meV}$, electron energies are the order of magnitude of $E\left(k_{B Z}\right)$. Therefore, both in one-temperature and two-temperature model, electron energies are not higher than $E\left(k_{B Z}\right)$, thus confirming the description with one band in the first Brillouin zone.

Assuming the electron distribution function $f_{e}$ can be only modified by collisions, the Boltzmann equation reads in the relaxation time approximation $\partial_{t} f_{e}=-\left(f_{e}-f_{\vec{e}}^{0}\right) / \tau(\vec{k})^{\sqrt{30}} \cdot f_{e}^{0}$ is the unperturbed distribution function and $\tau(\vec{k})$ is the relaxation time defined as:

$$
\frac{1}{\tau(\vec{k})}=v(\vec{k})=\frac{V}{8 \pi^{3}} \int\left[S^{-}\left(\vec{k}, \vec{k}^{\prime}\right)+S^{+}\left(\vec{k}, \vec{k}^{\prime}\right)\right] \mathrm{d} \vec{k}^{\prime}
$$

The term $S^{ \pm}\left(\vec{k}, \vec{k}^{\prime}\right)$ corresponds to the electron transition rate from a state $\vec{k}$ to a state $\vec{k}^{\prime}$ by absorbing (-) or emitting (+) a phonon of momentum $\vec{q}$. It is given by the Fermi golden rule 53 :

$$
S^{ \pm}\left(\vec{k}, \vec{k}^{\prime}\right)=\frac{2 \pi}{\hbar}\left|\left\langle\vec{k}^{\prime}\left|H_{e-p h}^{ \pm}\right| \vec{k}\right\rangle\right|^{2} \delta\left(E_{\vec{k}^{\prime}}-E_{\vec{k}} \mp \hbar \omega_{\beta}(q)\right)
$$

with $E_{\vec{k}}$ and $E_{\vec{k}}$, the energies of the states $\vec{k}$ and $\vec{k}^{\prime}$ respectively and $\omega_{\beta}(q)$ the frequency of the $\beta$-mode phonon. If the umklapp processes are neglected, matrix elements read $[3$ :

$$
\begin{aligned}
\left\langle\vec{k}^{\prime}\left|H_{e-p h}^{ \pm}\right| \vec{k}\right\rangle= & \left(\frac{\hbar}{2 \rho V \omega_{\beta}(q)}\right)^{1 / 2}\left(g_{\beta}+\frac{1}{2} \pm \frac{1}{2}\right)^{1 / 2} \\
& \times \mathscr{I}_{\vec{q}, p}\left(\vec{k}, \vec{k}^{\prime}\right) \delta\left(\vec{k}^{\prime}-\vec{k} \pm \vec{q}\right)
\end{aligned}
$$

which depends on the density $\rho$, the volume $V$ and the lattice temperature $T_{i l}$ through the Bose-Einstein distribution $g_{\beta}=\left[\exp \left(\hbar \omega_{\beta}(q) / k_{B} T_{i l}\right)-1\right]^{-1} . \quad \delta\left(\vec{k}^{\prime}-\vec{k} \pm \vec{q}\right)$ represents the momentum conservation and $\mathscr{I}_{\vec{q}, p}\left(\vec{k}, \vec{k}^{\prime}\right)$ the coupling between the lattice potential and the electronic states. It is calculated in the deformation potential approximation as a compromise between a simple model and good results leading to 53 :

$$
\mathscr{I}_{\vec{q}, p}\left(\vec{k}, \vec{k}^{\prime}\right)=i \vec{q} \cdot \vec{e}_{\vec{q}, p} \mathscr{U}\left(q, \omega_{\beta}(q)\right)
$$

The potential $\mathscr{U}\left(q, \omega_{\beta}(q)\right)$ depends on whether an acoustic or an optical phonon is considered. For an acoustic phonon, it is constant equal to a few $\mathrm{eV}$ in the limit of small momentum transfer and one has $\mathscr{U}=\delta \mathscr{E} / \Delta$. It corresponds to a measure of the deformation potential $\delta \mathscr{E}$ due to the lattice dilatation $\Delta$ induced by the acoustic mode ${ }^{53}$. Neither $\mathscr{U}$ nor $\delta \mathscr{E}$ nor $\Delta$ can be evaluated simply for dielectrics. Based on cross section evaluation, Fishcetti et al. ${ }^{[2]}$ set $\mathscr{U} \approx 3.5 \mathrm{eV}$ for $\mathrm{SiO}_{2}$, that is around $40 \%$ of the band gap energy $E_{g}$. By analogy with metals where the deformation potential is proportional to the Fermi energy, it is assumed that the deformation potential of dielectric materials is proportional to the band gap. With $E_{g}=4.05 \mathrm{eV}^{\sqrt{55}}$ for polystyrene, this leads to $\mathscr{U}=1.62 \mathrm{eV}$. This ensures to obtain the standard order of magnitude of a few $\mathrm{eV}$ for the deformation potentia 53 . For an optical phonon, it is given by the Fröhlich formalism 53 |56|57.

$$
\mathscr{U}\left(q, \omega_{\beta}(q)\right)=-\left(4 \pi e^{2} \rho \omega_{\beta}^{2}(\vec{q})\left(\frac{1}{\varepsilon_{r}^{\infty}}-\frac{1}{\varepsilon_{r}^{0}}\right)\right)^{1 / 2} \frac{1}{\vec{q}^{2}}
$$

where $\varepsilon_{r}^{0}$ and $\varepsilon_{r}^{\infty}$ are the dielectric constants at low and high frequencies respectively. The momentum conservation equation giving $\vec{k}^{\prime 2}=\vec{k}^{2}+\vec{q}^{2} \pm 2 k q \cos (\theta)$, it is possible to write the term representing the energy conservation as:

$$
\begin{aligned}
& \delta\left(E_{\vec{k}^{\prime}}-E_{\vec{k}} \mp \hbar \omega_{\beta}(q)\right) \\
& \quad=\frac{m^{*}}{\hbar^{2} k q} \delta\left( \pm \cos (\theta)+\frac{q}{2 k} \mp \frac{m^{*} \omega_{\beta}(q)}{\hbar k q}\right)
\end{aligned}
$$

where the parabolic band approximation has been used $E_{\vec{k}^{\prime}}=$ $\hbar^{2} \vec{k}^{2} / 2 m^{*}$ with $m^{*}$ the effective electron mass assumed to be equal to the free electron mass $m_{e}$. Finally:

$$
\begin{aligned}
& S^{ \pm}\left(\vec{k}, \vec{k}^{\prime}\right)=\frac{\pi q m^{*} \mathscr{U}^{2}\left(q, \omega_{\beta}(q)\right)}{\hbar^{2} \rho V \omega_{\beta}(q)}\left(g_{\beta}+\frac{1}{2} \pm \frac{1}{2}\right) \\
& \quad \times \delta\left( \pm \cos (\theta)+\frac{q}{2 k} \mp \frac{m^{*} \omega_{\beta}(q)}{\hbar k q}\right)
\end{aligned}
$$

It appears $S^{ \pm}\left(\vec{k}, \vec{k}^{\prime}\right)$ depends only on $q$ and $\cos \theta$ and Eq. (A1) becomes

$v(\vec{k})=\frac{V}{4 \pi^{3}} \int_{0}^{\infty} \int_{-1}^{1}\left(S^{-}\left(\vec{k}, \vec{k}^{\prime}\right)+S^{+}\left(\vec{k}, \vec{k}^{\prime}\right)\right) q^{2} \mathrm{~d} q \mathrm{~d} \cos (\theta)$

Assuming $q \rightarrow 0$, this leads to a linear relation dispersion for acoustic phonon: $\omega_{L A}(\vec{q}) \approx c_{s} q$, and constant dispersion relation for optical phonon: $\omega_{L O}(\vec{q}) \approx \omega_{L O}$. The demonstration of these approximations is given in Appendix B. Finally, plugging Eq. (A7) and (A8), this leads to the following collision frequency for acoustic phonons 28 :

$$
v_{A C}(\vec{k})=\frac{\mathscr{U}^{2} k_{B} T_{i l} m^{*}}{2 \pi \rho c_{s}^{2} \hbar^{3} k}\left(k^{2}+\left(\frac{m^{*} c_{s}}{\hbar}\right)^{2}\right)
$$

where it has been supposed the temperature is much higher than the phonon energy leading to a simplified phonon distribution: $n_{A C} \approx n_{A C}+1 \approx k_{B} T_{i l} / \hbar c_{s} q$. For optical phonons one 
has:

$$
\begin{aligned}
& v_{O P}(\vec{k})=\frac{e^{2} m^{*} \omega_{L O}}{\hbar^{2} k}\left(\frac{1}{\varepsilon_{r}^{\infty}}-\frac{1}{\varepsilon^{0}}\right) \times \\
& \left(n_{L O} \ln \left(\frac{\sqrt{1+2 m^{*} \omega_{L O} / \hbar k^{2}}+1}{\sqrt{1+2 m^{*} \omega_{L O} / \hbar k^{2}}-1}\right)\right. \\
& \left.+\left(n_{L O}+1\right) \ln \left(\frac{1+\sqrt{1-2 m^{*} \omega_{L O} / \hbar k^{2}}}{1-\sqrt{1-2 m^{*} \omega_{L O} / \hbar k^{2}}}\right)\right)
\end{aligned}
$$

\section{Appendix B: Polystyrene dispersion relation}

The polystyrene consists of a chain of the unit cell $\mathrm{CH}(\mathrm{Ph})-\mathrm{CH}_{2}$ where $\mathrm{Ph}$ refers to the phenyl group $\mathrm{C}_{6} \mathrm{H}_{5}$ (see Fig. 1). Even if it is not always possible to define a periodicity from a macroscopic point view, it can be defined a local periodicity of the unit cell $\mathrm{CH}(\mathrm{PH})-\mathrm{CH}_{2}$ in order to get the phonon relation dispersion. However, the unit cell is here composed of 16 atoms leading to 48 eigenmodes in three dimensions. In order to get a problem easier to handle, only phonons propagating along the carbon chain, which is assumed linear, are considered in a first approximation. This allows us to consider a one dimension problem. In addition, the unit cell is also simplified by assuming it is composed of the phenyl group with a mass $m_{P h}=6 m_{C}+5 m_{H}$, the group $\mathrm{CH}$ with a mass $m_{\mathrm{CH}}=m_{C}+m_{H}$ and the group $\mathrm{CH}_{2}$ with a mass $m_{\mathrm{CH}_{2}}=m_{C}+2 m_{H}$. This simplified form of the unit cell is given by Fig. 10 .

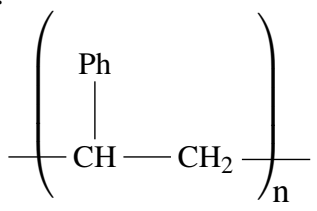

FIG. 10. Simplified structure of the unit cell of polystyrene

Within this simplification, one acoustic mode and two optical modes are expected. Finally, it is assumed only the closest neighbors interact among themselves and the interaction potential is taken in the harmonic approximation, i.e. $U(x)=k x^{2} / 2$ with $k$ the interaction energy determined experimentally. This leads to :

$$
\begin{aligned}
U_{T O T} & =U_{\mathrm{Ph}}+U_{\mathrm{CH}}+U_{\mathrm{CH}_{2}} \\
U_{\mathrm{Ph}-\mathrm{CH}} & =\frac{G}{2} \sum_{n}\left[u_{\mathrm{Ph}}\left(n a, d_{1}\right)-u_{\mathrm{CH}}(n a, 0)\right]^{2} \\
U_{\mathrm{CH}-\mathrm{CH}_{2}} & =\frac{K}{2} \sum_{n}\left[u_{\mathrm{CH}}(n a, 0)-u_{\mathrm{CH}_{2}}(n a, 0)\right]^{2} \\
U_{\mathrm{CH}_{2}-\mathrm{CH}} & =\frac{K}{2} \sum_{n}\left[u_{\mathrm{CH}_{2}}(n a, 0)-u_{\mathrm{CH}}((n+1) a, 0)\right]^{2}
\end{aligned}
$$

The constants $G$ and $K$ characterize the $\mathrm{CH}-\mathrm{Ph}$ and $\mathrm{CH}-$ $\mathrm{CH}_{2}$ bonds respectively. They are defined in terms of the

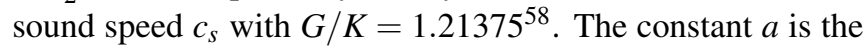
$\mathrm{CH}-\mathrm{CH}_{2}$ bond length, and quantities $u_{X}(x, y)$ represent the displacement of the group $X$ having the initial position $x$ and $y$.
The motion equations are given by the Euler-Lagrange equations :

$$
\begin{aligned}
m_{\mathrm{Ph}} \frac{\partial^{2} u_{\mathrm{Ph}}\left(n a, d_{1}\right)}{\partial t^{2}} & =-\frac{\partial U_{T O T}}{\partial u_{\mathrm{Ph}}\left(n a, d_{1}\right)} \\
m_{\mathrm{CH}} \frac{\partial^{2} u_{\mathrm{CH}}(n a, 0)}{\partial t^{2}} & =-\frac{\partial U_{T O T}}{\partial u_{\mathrm{CH}}(n a, 0)} \\
m_{\mathrm{CH}_{2}} \frac{\partial^{2} u_{\mathrm{CH}_{2}}(n a, 0)}{\partial t^{2}} & =-\frac{\partial U_{T O T}}{\partial u_{\mathrm{CH}_{2}}(n a, 0)}
\end{aligned}
$$

By seeking for solutions in the form $u_{X}(x, y)=$ $U_{X} e^{i\left(k_{x} x+k_{y} y-\omega t\right)}$ with $U_{X}$ the amplitude, this leads to a linear system that has a solution if and only if :

$$
\left|\begin{array}{ccc}
\left(G-m_{\mathrm{Ph}} \omega^{2}\right) e^{i k_{y} d_{1}} & -G & 0 \\
-G e^{i k_{y} d_{1}} & G+2 K-m_{\mathrm{CH}} \omega^{2} & -K\left(1+e^{-i k_{x} a}\right) \\
0 & -K\left(1+e^{i k_{x} a}\right) & 2 K-m_{\mathrm{CH}_{2}} \omega^{2}
\end{array}\right|=0
$$

It appears the term $e^{i k_{y} d_{1}}$ vanishes, because only longitudinal modes are considered. The nullity of the determinant leads to a 6th-order polynomial equation:

$$
\alpha \omega^{6}+\beta \omega^{4}+\gamma \omega^{2}+\delta=0
$$

where the coefficients $\alpha, \beta, \gamma$ and $\delta$ are given by:

$$
\begin{aligned}
\alpha= & -m_{\mathrm{Ph}} m_{\mathrm{CH}} m_{\mathrm{CH}_{2}} \\
\beta= & G m_{\mathrm{CH}_{2}}\left(m_{\mathrm{CH}}+m_{\mathrm{Ph}}\right)+2 K m_{\mathrm{Ph}}\left(m_{\mathrm{CH}}+m_{\mathrm{CH}_{2}}\right)(\mathrm{B} 1 \\
\gamma= & {\left[4 K^{2} \cos ^{2}\left(\frac{k_{x} a}{2}\right)-2 K(2 K+G)\right] m_{\mathrm{Ph}} } \\
& -2 G K\left(m_{\mathrm{CH}}+m_{\mathrm{CH}_{2}}\right) \\
\delta= & 4 G K^{2}\left[1-\cos ^{2}\left(\frac{k_{x} a}{2}\right)\right]
\end{aligned}
$$

Finally, the eigenmodes of the polystyrene are given by :

$$
\begin{gathered}
\omega_{m}^{2}=z_{m}-\frac{\beta}{3 \alpha}, m \in\{0,1,2\} \\
z_{m}=2 \sqrt{-\frac{p}{3}} \cos \left[\frac{1}{3} \arccos \left(\frac{3 q}{2 p} \sqrt{-\frac{3}{p}}\right)+\frac{2(m+1) \pi}{3}\right]
\end{gathered}
$$

with

$$
\begin{aligned}
& p=-\frac{\beta^{2}}{3 \alpha^{2}}+\frac{\gamma}{\alpha} \\
& q=\frac{2 \beta^{3}}{27 \alpha^{3}}-\frac{9 \beta \gamma}{27 \alpha^{2}}+\frac{\delta}{\alpha}
\end{aligned}
$$

Figure 11 plots these three modes. It is observed one acoustic mode and two optical modes as expected. The acoustic and optical modes are characterized in the limit of $q \rightarrow 0$ by a linear and constant behavior respectively. It is found that phonon energies lie in the range of other dielectrics, as $\mathrm{SiO}_{2}{ }^{5960}$. 


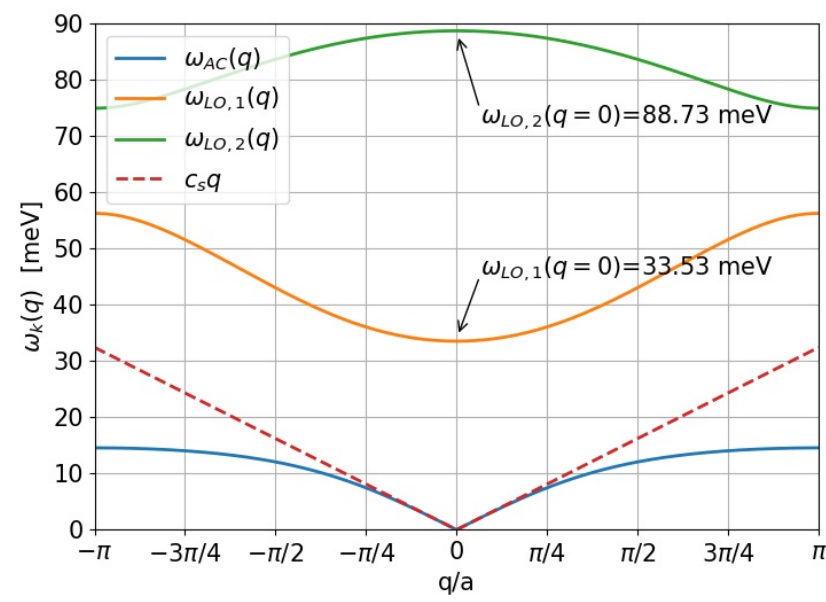

FIG. 11. Polystyrene eigenmodes

\section{Appendix C: Approximation of the chemical potential}

In the free electron gas model, the electron density is defined by:

$$
n_{e}=\int_{0}^{+\infty} \frac{\sqrt{2} m_{e}^{3 / 2}}{\hbar^{3} \pi^{2}} \frac{\sqrt{\varepsilon}}{e^{(\varepsilon-\mu) / k_{B} T_{e}}+1} \mathrm{~d} \varepsilon
$$

where the bottom of the conduction band is set to zero. By defining the two following dimensionless parameters

$$
x=\frac{\mu}{k_{B} T_{e}} \quad ; \quad y=\frac{\hbar^{3} \pi^{2} n_{e}}{\sqrt{2}\left(m_{e} k_{B} T_{e}\right)^{3 / 2}}
$$

it is possible to write Eq. (C1) in such a way that the FermiDirac integral $F_{1 / 2}$ appears:

$$
y=\int_{0}^{+\infty} \frac{\sqrt{t}}{e^{t-x}+1}=F_{1 / 2}(x)
$$

As a consequence, looking for an expression of the chemical potential becomes equivalent to find the inverse function of $F_{1 / 2}$. This is done by taking approximations of $F_{1 / 2}$ in the two asymptotic cases 6162 .

$$
\begin{aligned}
& x<1.13 \Leftrightarrow y<1.52 \Rightarrow F_{1 / 2}(x)=\frac{\sqrt{\pi}}{0.5+2 e^{-x}} \\
& x>1.13 \Leftrightarrow y>1.52 \Rightarrow F_{1 / 2}(x)=\frac{2}{3}\left(x^{2}+\frac{\pi^{2}}{6}\right)^{3 / 4}
\end{aligned}
$$

Because these approximations lead to a discontinuity for $x=$ $1.13, y=1.52$, it is possible to get a continuous approximation by weighting the previous two expressions with the logistic function $V\left(y, y_{0}, k\right)=\left[1+e^{-a\left(y-y_{0}\right)}\right]^{-1}$ around these values. Thus, the following approximation of the chemical potential is deduced:

$$
\begin{gathered}
y<1: \mu \approx k_{B} T_{e} \ln \left(\frac{4 y}{2 \sqrt{\pi}-y}\right) \\
1<y<2.5: \mu \approx k_{B} T_{e}\left\{\frac{\ln [4 y /(2 \sqrt{\pi}-y)]}{1+e^{a\left(y-y_{0}\right)}}\right. \\
\left.+\frac{\sqrt{(3 y / 2)^{4 / 3}-\pi^{2} / 6}}{1+e^{-a\left(y-y_{0}\right)}}\right\} \\
y>2.5: \mu \approx k_{B} T_{e} \sqrt{\left(\frac{3 y}{2}\right)^{4 / 3}-\frac{\pi^{2}}{6}}
\end{gathered}
$$

with the parameters chosen as $y_{0}=1.52$ and $a=15$ in order to have a good approximation in the transition zone.

\section{Appendix D: Ionization}

Saha equations are used in order to describe ionization of styrene, benzene and acetylene because it has already been shown valid for ICF conditions 44 . They were originally established assuming thermal equilibrium $T_{e}=T_{i l}$ but it is possible to establish modified Saha equations for $T_{e} \neq T_{i l}$ as follows 63 .

$$
\frac{n_{j+1} n_{e}}{n_{j}}=2\left(\frac{2 \pi m_{e} k_{B} T_{e}}{h^{2}}\right)^{3 / 2} \frac{Q_{j+1}\left(T_{e}\right)}{Q_{j}\left(T_{e}\right)} \exp \left(-\frac{U_{j+1}}{k_{B} T_{e}}\right)
$$

where $U_{j+1}$ is the $(j+1)$ th ionization potential, $n_{j}$ and $n_{j+1}$ are the densities of atoms ionized $j$ and $j+1$ times respectively, and $Q_{j}\left(T_{e}\right)$ and $Q_{j+1}\left(T_{e}\right)$ are their partition functions reduced to the translational, rotational and vibrational contributions. As a first approximation, the rotational and vibrational parts can be neglected leading to the ratio $Q_{j+1}\left(T_{e}\right) / Q_{j}\left(T_{e}\right)$ equal to unity. Because the mass of the atoms ionized $j$ and $j+1$ times can be considered as the same, the translational partition functions cancel. Note that the prefactor 2 corresponds to the electron spin degeneracy. This equation involves three densities and the problem is closed with the two following relations:

$$
n_{e}=\sum_{j=1}^{N} Z_{j} n_{j}=\sum_{j=1}^{N} j n_{j} \quad ; \quad n_{T}=n_{n}+\sum_{j=1}^{N} n_{j}
$$

$N$ is the number of ionized states taken into account. In an exact description, it is equal to the number of electrons of the particle. $Z_{j}=j$ and $n_{j}$ are the charge state and the density of particles ionized $j$ times respectively. $n_{n}$ is the density of neutrals and $n_{T}$ is the total density. The averaged ionization state $\langle Z\rangle$ is given by:

$$
<Z>=\frac{n_{e}}{n_{T}}
$$

The ionization model of the carbon hydrogen mixture is obtained by ab initio calculations and the averaged ionization state $\langle Z>$ is given by 4 :

$$
<Z>=\frac{\xi}{2}\left(\sqrt{1+\frac{4}{\xi}}-1\right) Z_{\text {max }}
$$


with $Z_{\max }=\left(Z_{H}+Z_{C}\right) / 2=3.5$ and:

$$
\xi=\frac{\alpha_{0}}{n_{i}}\left(\frac{2 \pi m_{e} k_{B} T}{h^{2}}\right)^{3 / 2} \exp \left(-\frac{f_{Z}}{k_{B} T}\right)
$$

while the average-ionization potential is given by:

$$
\begin{aligned}
f_{Z}= & \alpha_{1}+\alpha_{2} k_{B} T\left[\left(1+\sqrt{3 \Gamma_{0}}\right)^{1 / 4}-1\right]+\alpha_{3}\left(k_{B} T\right)^{0.9} \\
& +\left(\frac{\alpha_{4}}{r_{0}}+\frac{\alpha_{5}}{r_{0}^{2}}+\frac{\alpha_{6}}{r_{0}^{3}}\right) k_{B} T
\end{aligned}
$$

with $r_{0}=a_{i} / a_{B}$ the ion-ion interdistance defined as the ratio of the ion sphere radius $a_{i}=\left(3 / 4 \pi n_{i}\right)^{1 / 3}$ over the Bohr radius $a_{B}, \Gamma_{0}=1 / r_{0} k_{B} T$ the ion-ion coupling parameter in atomic units, and $\alpha_{i}$ fitting parameters: $\alpha_{0}=87.222, \alpha_{1}=10.866$, $\alpha_{2}=-28.412, \alpha_{3}=17.915, \alpha_{4}=-2.422, \alpha_{5}=0.595$ and $\alpha_{6}=-2.369 \times 10^{-2}$. Finally, the electron, ion and neutral densities $n_{e}, n_{i}$ and $n_{0}$ are given by:

$$
n_{e}=<Z>n_{T} \quad ; \quad n_{i}=\min \left(n_{e}, n_{T}\right) \quad ; \quad n_{0}=n_{T}-n_{i}
$$

Note that defining $n_{i}$ by this way does not allow to access to densities of each ion existing $n_{H^{+}}, n_{C^{+}}, n_{C^{2+}}$, etc. As the rigorous relation linking the electron density and the density of each charge state is given by Eq. (D2), if $n_{e}$ is known, it is impossible to deduce each ion density $n_{j}$.

${ }^{1}$ J. Nuckolls, L. Wood, A. Thiessen, and G. Zimmerman, Nature 239, 139 (1972).

${ }^{2}$ D. D. Meyerhofer, R. L. McCrory, R. Betti, T. R. Boehly, D. T. Casey, T. J. B. Collins, R. S. Craxton, J. A. Delettrez, D. H. Edgell, R. Epstein, K. A. Fletcher, J. A. Frenje, Y. Y. Glebov, V. N. Goncharov, D. R. Harding, S. X. Hu, I. V. Igumenshchev, J. P. Knauer, C. K. Li, J. A. Marozas, F. J. Marshall, P. W. McKenty, P. M. Nilson, S. P. Padalino, R. D. Petrasso, P. B. Radha, S. P. Regan, T. C. Sangster, F. H. Séguin, W. Seka, R. W. Short, D. Shvarts, S. Skupsky, J. M. Soures, C. Stoeckl, W. Theobald, and B. Yaakobi, Nuclear Fusion 51, 053010 (2011)

${ }^{3}$ V. N. Goncharov, T. C. Sangster, R. Betti, T. R. Boehly, M. J. Bonino, T. J. B. Collins, R. S. Craxton, J. A. Delettrez, D. H. Edgell, R. Epstein, R. K. Follett, C. J. Forrest, D. H. Froula, V. Yu. Glebov, D. R. Harding, R. J. Henchen, S. X. Hu, I. V. Igumenshchev, R. Janezic, J. H. Kelly, T. J. Kessler, T. Z. Kosc, S. J. Loucks, J. A. Marozas, F. J. Marshall, A. V. Maximov, R. L. McCrory, P. W. McKenty, D. D. Meyerhofer, D. T. Michel, J. F. Myatt, R. Nora, P. B. Radha, S. P. Regan, W. Seka, W. T. Shmayda, R. W. Short, A. Shvydky, S. Skupsky, C. Stoeckl, B. Yaakobi, J. A. Frenje, M. GatuJohnson, R. D. Petrasso, and D. T. Casey, Physics of Plasmas 21, 056315 (2014)

${ }^{4}$ R. Craxton, K. Anderson, T. Boehly, V. Goncharov, D. Harding, J. Knauer, M. McCrory, P. McKenty, D. Meyerhofer, J. Myatt, A. Schmitt, J. Sethian, R. Short, S. Skupsky, W. Theobald, W. Kruer, K. Tanaka, R. Betti, T. Collins, J. Delettrez, S. Hu, J. Marozas, A. Maximov, D. Michel, P. Radha, S. Regan, T. Sangster, W. Seka, A. Solodov, J. S. ans C. Stoeckl, and J. Zuegel, Phys. Plasmas 22, 110501 (2015).

${ }^{5}$ G. Duchateau, S. X. Hu, A. Pineau, A. Kar, B. Chimier, A. Casner, V. Tikhonchuk, V. N. Goncharov, P. B. Radha, and E. M. Campbell, Phys. Rev. E 100, 033201 (2019).

${ }^{6}$ R. Ishizaki and K. Nishihara, Phys. Rev. Lett. 78, 1920 (1997).

${ }^{7}$ S. Bodner, Phys. Rev. Lett. 33, 761 (1974)

${ }^{8}$ P. B. Radha, V. N. Goncharov, T. J. B. Collins, J. A. Delettrez, Y. Elbaz, V. Y. Glebov, R. L. Keck, D. E. Keller, J. P. Knauer, J. A. Marozas, F. J. Marshall, P. W. McKenty, D. D. Meyerhofer, S. P. Regan, T. C. Sangster, D. Shvarts, S. Skupsky, Y. Srebro, R. P. J. Town, and C. Stoeckl, Physics of Plasmas 12, 032702 (2005).
${ }^{9}$ S. X. Hu, P. B. Radha, J. A. Marozas, R. Betti, T. J. B. Collins, R. S. Craxton, J. A. Delettrez, D. H. Edgell, R. Epstein, V. N. Goncharov, I. V. Igumenshchev, F. J. Marshall, R. L. McCrory, D. D. Meyerhofer, S. P. Regan, T. C. Sangster, S. Skupsky, V. A. Smalyuk, Y. Elbaz, and D. Shvarts, Physics of Plasmas 16, 112706 (2009).

${ }^{10}$ J. L. Peebles, S. X. Hu, W. Theobald, V. N. Goncharov, N. Whiting, P. M. Celliers, S. J. Ali, G. Duchateau, E. M. Campbell, T. R. Boehly, and S. P. Regan, Phys. Rev. E 99 (2019).

${ }^{11}$ L. Spitzer, Physics of Fully Ionized Gases (Interscience Publishers Inc., 1956).

${ }^{12}$ J. Mark, Polymer Data Handbook (Oxford University Press, Inc., 1999).

${ }^{13}$ D. Feldmann, J. Kutzner, J. Laukemper, S. MacRobert, and K. Welge, Appl. Phys. B 44, 81 (1987).

${ }^{14}$ H. Fukumura, N. Mibuka, S. Eura, and H. Masuhara, J. Phys. Chem. 97, 13761 (1993)

${ }^{15}$ M. Tsunekawa, S. Nishio, and H. Sato, J. Appl. Phys 76, 5598 (1994).

${ }^{16}$ Y. Tsuboi, S. Sakashita, H. Hatanaka, H. Fukumura, and H. Masuahara, Laser Chem. 16, 167 (1996).

${ }^{17}$ J. Franklin and S. Caroll, J. Am. Chem. Soc. 91, 5940 (1969).

${ }^{18}$ R. Dunbar, J. Am. Chem. Soc. 111, 5572 (1989).

${ }^{19}$ R. Dunbar, J. Phys. Chem. (1990).

${ }^{20} \mathrm{P}$. Flowers, K. Theopold, and R. Langley, Chemistry (Rice University, 2015).

${ }^{21}$ J. McMurry, R. Fay, and J. Robinson, Chemistry (Pearson, 2015).

${ }^{22}$ Y. Luo, Comprehensive Handbook of Chemical Bond Energies (CRC Press, 2007).

${ }^{23}$ K. Ervin, S. Gronert, S. Barlow, M. Gilles, A. Harrison, V. Bierbaum, C. DePuy, W. Lineberger, and G. Ellison, J. Am. Chem. Soc. 112, 5750 (1990).

${ }^{24}$ S. Blanksby and G. B. Ellison, Acc. Chem. Res 36, 255 (2003)

${ }^{25}$ D. Baldwin, M. Buntime, and D. Chandler, J. Chem. Phys. 93, 6578 (1990).

${ }^{26} \mathrm{~J}$. McMurry, Organic Chemistry, 9th ed. (Cengage Learning, 2016).

${ }^{27}$ J. Bandrup, E. Immergut, and E. Grulke, Polymer Handbook, fourth edition ed. (Wiley-Blackwell, 1998).

${ }^{28}$ M. Fischetti, D. DiMaria, S. Brorson, T. Theis, and J. Kirtley, Phys. Rev. B 31, 8124 (1985).

${ }^{29}$ G. Zhao, M. Mors, T. Wenckebach, and P. Planken, J. Opt. Soc. Am. B 19, 061476 (2002).

${ }^{30}$ N. Ashcroft and N. Mermin, Physique des Solides (EDP Sciences, 2002).

${ }^{31}$ W. Smith, J. Bechtel, and N. Bloembergen, Phys. Rev. B 12, 706 (1975).

${ }^{32}$ D. Arnold, E. Cartier, and D. DiMaria, Phys. Rev. B 49, 10278 (1994).

${ }^{33} \mathrm{M}$. Al-Joboury and D. Turner, J. Chem. Soc. , 4434 (1963).

${ }^{34} \mathrm{U}$. Inan and M. Golkowski, Principles of Plasma Physics for Engineers and Scientists (Cambridge University Press, 2011).

${ }^{35}$ C. Ordonnez and M. Molina, Phys. Plasmas 1, 2515 (1994).

${ }^{36}$ L. Landau and E. Lifchitz, Physique Théorique : Mécanique, 5th ed. (MirEllipses, 1994).

${ }^{37}$ T. Ramazanov and K. Dzhumagulova, Phys. Plasmas.

${ }^{38}$ Y. Lee and R. More, Phys Fluids 27 (1984).

${ }^{39}$ H. Brysk, P. Campbell, and P. Hammerling, Plasma Phys. 17, 473 (1975).

${ }^{40}$ F. Olver, D. Lozier, R. Boisvert, and C. Clarck, NIST Handbook of Mathematical Functions (Cambridge University Press, New York, 2010).

${ }^{41}$ J. D. Huba, NRL Plasma Formulary (U.S. GPO, Washington, D. C., 2016).

${ }^{42}$ L. D. Landau and E. M. Lifshitz, Quantum Mechanics (Pergamon, NewYork, 1965)

${ }^{43}$ D. Gericke, M. Murillo, and M. Schlanges, Phys. Rev. E 65, 036418 (2002).

${ }^{44}$ S. X. Hu, L. A. Collins, V. N. Goncharov, J. D. Kress, R. L. McCrory, and S. Skupsky, Phys. Plasmas 23, 042704 (2016).

${ }^{45}$ J. Delcroix and A. Bers, Physique des Plasmas I, Vol. 1 (CNRS Editions and EDP Sciences, 1994)

${ }^{46}$ V. Silin, JETP 20, 1510 (1965).

${ }^{47}$ T. Bornath, M. Schlanges, P. Hilse, and D. Kremp, Phys. Rev. E 64 (2001).

${ }^{48}$ G. R. A. Wierling, Th. Millat and R. Redmer, Phys. Plasmas 8, 3310 (2001).

${ }^{49}$ T. B. P. Hilse, M. Schlanges and D. Kremp, Phys. Rev. L 71, 056408 (2005).

${ }^{50}$ A. Grinenko and D. Gericke, Phys. Rev. Lett. 103 (2009).

${ }^{51}$ S. Anderson and E. Freeman, J. Polym. Sci. 54, 253 (1961).

${ }^{52}$ P. Mora, "Private communication,".

${ }^{53}$ J. Ziman, Electrons and Phonons, edited by N. Mott, E. Bullard, and D. Wilkinson (Clarendon Press, 1960). 
${ }^{54}$ M. Mondello, H. Yang, H. Furuya, and R. Roe, Macromolecules 27, 3566 (1994).

${ }^{55}$ V. Sangawar and M. Golchha, Int. Jour. Sci. Eng. Res. 4, 2700 (2013).

${ }^{56}$ H. Fröhlich, H. Pelzer, and S. Zienau, Philosophical Magazine Series 741 , 221 (1950).

${ }^{57}$ F. Guistino, Rev. Mod. Phys. (2017).

${ }^{58}$ S. Rastogi and V. Gupta, Journal of Macromolecular Science, Part B:
Physics 33, 129 (1994).

${ }^{59}$ I. Godmanis, A. Trukhin, and K. Hübner, Phys. Stat. Sol. B 116, 279 (1983).

${ }^{60}$ F. Quéré, S. Guizard, P. Martin, G. Petite, H. Merdji, B. Carré, J.-F. Hergott, and L. Le Déroff, Phys. Rev. B 61, 9883 (2000).

${ }^{61}$ L. Soonckindt and L. Lassabatere, Rev. Phys. Appl. 13, 581 (1978).

${ }^{62} \mathrm{~S}$. Li, Semiconductor Physical Electronics (Springer, 2006).

${ }^{63}$ X. Chen and P. Han, J. Phys. D: Appl. Phys. 32, 1711 (1999). 